\title{
Prescrição de medicamentos para idosos: indicadores de qualidade, seus instrumentos e medidas como estratégias indispensáveis no sistema de saúde
}

\author{
Prescription of medicines for the aged: quality indicators, their instruments and measures as \\ indispensable strategies in the health system \\ Prescripción de medicamentos para los ancianos: Indicadores de calidad, sus instrumentos y \\ medidas como estrategias indispensables en el sistema de salud
}

\author{
Mônica de Souza Brito Conti \\ ORCID: https://orcid.org/0000-0002-4810-7566 \\ Universidade Federal de São Paulo, Brasil \\ E-mail: msbconti@unifesp.br \\ Adriana Sañudo \\ ORCID: https://orcid.org/0000-0003-1187-0143 \\ Universidade Federal de São Paulo, Brasil \\ E-mail: adriana.sanudo@unifesp.br \\ Luiz Roberto Ramos \\ ORCID: https://orcid.org/0000-0003-3143-8315 \\ Universidade Federal de São Paulo, Brasil \\ E-mail: 1rramos1953@gmail.com
}

\begin{abstract}
Resumo
A terapia medicamentosa é parte integrante do plano de assistência ao idoso, sendo a prescrição um processo difícil e desafiador. Investigar a prescrição de idosos é uma prática preconizada como ferramenta na avaliação da qualidade do serviço de saúde, pois, o tratamento medicamentoso é um elemento essencial da atenção clínica ao idoso, e a otimização da prescrição se faz necessária, porquanto tem se tornado mundialmente um importante problema de saúde. O presente estudo tem como objetivo apresentar e discutir os indicadores de qualidade da prescrição em idosos e o uso de instrumentos e medidas como estratégias para sua apropriada utilização no sistema de saúde. O método utilizado foi a revisão narrativa da literatura, por meio de obras já publicadas, encontradas em bases de dados nos idiomas português e inglês, para colaborar com a educação continuada de profissionais de saúde e proporcionar o uso racional e seguro da terapêutica medicamentosa. Diante dos dados apresentados, é imprescindível promover uma prescrição de qualidade e adequada a fim de que os idosos possam ter uma melhor qualidade de vida para que vivam o maior tempo possível em seus domicílios com saúde, autonomia e independência.

Palavras-chave: Idosos; Prescrições; Uso de medicamentos; Prescrição inadequada; Indicadores de qualidade em assistência à saúde.
\end{abstract}

\begin{abstract}
Drug therapy is an integral part of the aged care plan, and prescribing is a difficult and challenging process. Investigating the prescription of the aged is a practice recommended as a tool in the evaluation of the quality of the health service, since drug treatment is an essential element of clinical care for the aged, and the optimization of prescription is necessary, as it has become a worldwide important health problem. The present study aims to present and discuss the quality indicators of prescription in the aged and the use of instruments and measures as strategies for their appropriate use in the health system. The method used was the narrative review of the literature, through works already published, found in databases in Portuguese and English, to collaborate with the continuing education of health professionals and provide the rational and safe use of drug therapy. In view of the data presented, it is essential to promote a quality and adequate prescription so that the aged can have a better quality of life so that they can live as long as possible in their homes with health, autonomy and independence.
\end{abstract}

Keywords: Aged; Prescriptions; Drug utilization; Inappropriate prescribing; Quality indicators in health care.

\section{Resumen}

La terapia con medicamentos es una parte integral del plan de cuidado de los ancianos, y la prescripción es un proceso difícil y desafiante. Investigar la prescripción del anciano es una práctica recomendada como herramienta en la evaluación de la calidad del servicio de salud, ya que el tratamiento farmacológico es un elemento esencial de la atención clínica al anciano, y la optimización de la prescripción es necesaria, como se ha convertido un problema de 
salud de importancia mundial. El presente estudio tiene como objetivo presentar y discutir los indicadores de calidad de la prescripción en ancianos y el uso de instrumentos y medidas como estrategias para su uso adecuado en el sistema de salud. El método utilizado fue la revisión narrativa de la literatura, a través de trabajos ya publicados, encontrados en bases de datos en portugués e inglés, para colaborar con la educación continua de los profesionales de la salud y propiciar el uso racional y seguro de la farmacoterapia. A la vista de los datos presentados, es fundamental promover una prescripción adecuada y de calidad para que las personas mayores puedan tener una mejor calidad de vida para que puedan vivir el mayor tiempo posible en sus hogares con salud, autonomía e independencia.

Palabras clave: Ancianos; Prescripciónes; Utilización de medicamentos; Prescripción inadecuada; Indicadores de calidad de la atención de salud.

\section{Introdução}

O envelhecer é uma realidade brasileira, contudo esse envelhecimento vêm sendo acompanhado de uma elevada prevalência de doenças crônicas não transmissíveis (DCNTs) e consequentemente, um aumento na utilização de medicamentos de forma contínua, apresentando uma combinação que produz um aumento na demanda por serviços de saúde, principalmente, os vinculados ao Sistema Único de Saúde (SUS); uma vez que implicam tratamentos mais prolongados, onerosos, com recuperação vagarosa e complexa, muitas vezes exigindo procedimentos de custos elevados e que envolvam tecnologias avançadas (Ramos, 2003; Marengoni, et al., 2011; Vasconcelos \& Gomes, 2012; Veras, 2009; Veras \& Oliveira, 2018; Malta, et al., 2017; Praxedes, et al., 2021; Mercadante, et al.,2021).

A terapia medicamentosa é parte integrante do plano de assistência ao idoso, sendo a prescrição um processo complexo e desafiador - uma vez que envolve determinar se um medicamento é indicado, optar pela melhor terapia, definir a dose e horário adequados para o estado fisiológico do idoso, além de verificar a efetividade e toxicidade; também é provocador, já que no envelhecimento há mudanças fisiológicas que podem transformar os parâmetros farmacocinéticos ${ }^{1}$ e farmacodinâmicos $^{2}$ dos medicamentos, alterando o efeito desejado do fármaco ou tornando os idosos mais propensos à toxicidade farmacológica e ao desenvolvimento de eventos adversos (Baldoni, et al.,2010; Rovers \& Currie, 2010; Baldoni, et al., 2011; Silva, et al., 2012; Carvalho, et al., 2012; Shah \& Hajjar, 2012; Correr \& Otuki, 2013; Brunton, et al., 2018).

Além disso, no momento da prescrição deve-se levar em conta a redução na capacidade funcional e cognitiva do idoso, que pode confundir a leitura dos rótulos dos medicamentos ou ter dificuldade de compreensão verbal e escrita da receita, levando a uma aversão do paciente em aderir à farmacoterapia, podendo ocorrer a progressão da doença ou falha do tratamento - tornando-se um desafio para o sistema de saúde (Diaz, 2002; Vuyk, 2003; Rocha, et al., 2008; Dimitrow, et al., 2011).

Assim, medicamentos prescritos podem resultar positivamente no controle das doenças, ou negativamente, quando prescritos de forma irracional/inadequada - 30\% dos idosos utilizam mais de um medicamento desnecessário à sua farmacoterapia, por exemplo; e estudos têm apontado uma prevalência entre $30 \%$ e $45 \%$ de idosos que receberam prescrições inadequadas, bem como entre $30 \%$ e $55 \%$ das reações adversas aos medicamentos (RAM) responsáveis por hospitalizações foram analisadas como evitáveis por uma prescrição adequada (Onder, et al., 2002; Onder, et al., 2003; Gurwitz, et al., 2003; Fialová, et al., 2005; Passarelli, et al., 2005; Wawruch, et al., 2008; Gallagher, et al., 2008; Scott \& Jayathissa, 2010; SousaMuñoz, et al., 2012; Kaufmann, et al., 2014) .

A exemplo, no Brasil nota-se um grande número de prescrições oriundas dos serviços públicos de saúde não estarem de acordo com as questões técnicas e legais - indispensáveis para uma competente dispensação e utilização correta dos medicamentos; desse modo, retroalimentam a demanda por serviços clínicos, comprometendo a relação custo/efetividade dos

\footnotetext{
${ }^{1}$ Farmacocinética é o caminho que o fármaco percorre no organismo, desde a sua administração, absorção, distribuição e metabolismo, até a sua excreção. Cada uma dessas fases pode afetar positiva ou negativamente a ação do fármaco, ou seja, o quanto de fato do fármaco, consegue chegar ao seu local de ação.

${ }^{2}$ Farmacodinâmica é definida pelo estudo dos efeitos bioquímicos e fisiológicos dos fármacos e seus mecanismos de ação, ou seja, refere-se as alterações que o fármaco provoca organismo.
} 
tratamentos, gerando maiores gastos aos agentes envolvidos e reduzindo a qualidade de vida dos pacientes (Teixeira \& Lefévre, 2001; Lyra Jr, et al., 2002; Lyra Jr, et al., 2004).

A investigação das prescrições para idosos é uma prática preconizada como ferramenta na avaliação da qualidade do serviço de saúde, pois o tratamento medicamentoso é um elemento essencial da atenção clínica ao idoso, e a otimização da prescrição se faz necessária, pois tem se tornado mundialmente um importante problema de saúde. Assim, é imprescindível implementar políticas, ações e estratégias de saúde para melhorar a qualidade da atenção à saúde do idoso, promovendo um serviço de saúde que saiba gerenciar o idoso com multimorbidades e polifarmácia, a fim de se ter um envelhecimento saudável (Pereira, et al., 2004; Lyra Jr, et al., 2004; Ribeiro, et al., 2005; Costa, et al., 2008; Baldoni, et al., 2010; Portela, et al., 2010; Franceschet-de-Sousa, et al., 2010; Praxedes, et al., 2011; Sousa-Muñoz, et al., 2012; Manso, et al., 2015; Silva, et al., 2018).

E não só, ao revisar a farmacoterapia do idoso, ou seja, analisar sua prescrição, para que seja adequada e de qualidade, é imprescindível se atentar para os indicadores de qualidade da prescrição, seus instrumentos e medidas de avaliação, como: o número de medicamentos empregados (polifarmácia), as RAM e possível ocorrência de cascata iatrogênica, as associações que possam provocar interações medicamentosas (IM) potencialmente perigosas, bem como a proporção dos fármacos contraindicados à essa faixa etária - Medicamentos inapropriados para idosos (MPII), principalmente anticolinérgicos, pois a não observação desses indicadores pode levar os idosos a desenvolverem Problemas relacionados a medicamentos (PRMs) ${ }^{3}$ que acarretam perda da autonomia e independência (Lyra Jr, et al., 2004; Ribeiro, et al., 2005; Castellar, et al., 2007; Correr, et al., 2007; Portela, et al., 2010; Correr, et al., 2011; Correr \& Otuki, 2013; Silva, et al., 2018).

Diante disso, a observação dos indicadores na prescrição, seus instrumentos e medidas, como ações e estratégias de interesse individual e coletivo tem a finalidade de otimizar a terapêutica medicamentosa, permitindo a adoção de medidas corretivas que impactam desde a seleção, aquisição, prescrição e dispensação dos medicamentos, favorecendo a qualidade da prescrição e garantindo o uso racional e seguro desses insumos para essa faixa etária especial. Desse modo, o artigo tem como objetivo apresentar e discutir os indicadores de qualidade da prescrição em idosos e o uso de instrumentos e medidas como estratégias para uma apropriada utilização no sistema de saúde.

\section{Metodologia}

Trata-se de uma análise crítica da literatura acadêmica na forma de uma revisão narrativa, sobre a prescrição de medicamentos para idosos - o uso de indicadores, seus instrumentos e medidas na busca por qualidade. De acordo com Rother (2007): "os artigos de revisão narrativa são publicações amplas, apropriadas para descrever e discutir o desenvolvimento ou o 'estado da arte' de um determinado assunto, sob ponto de vista teórico ou conceitual".

As revisões narrativas constituem, basicamente, da análise de literatura publicada em livros, artigos de revista impressas e/ou eletrônicas, e na interpretação e exame crítico pessoal do autor. Porém, essas revisões não necessitam informar as fontes de informações utilizadas, a metodologia para a busca das referências, nem os critérios utilizados na avaliação e seleção dos trabalhos consultados. Assim, pesquisadores reforçam a ideia que, apesar da revisão narrativa não trazer a mesma rigidez metodológica de uma revisão integrativa ou sistemática, é necessário que ela traga conhecimentos suficientes que possam esclarecer sobre a importância e produção a respeito do assunto estudado, pois as revisões narrativas contribuem ao debate de determinadas temáticas, levantando questões e cooperando na aquisição e atualização do conhecimento em curto espaço de tempo (Rother, 2007; Casarin, et al., 2020).

O presente estudo foi realizado a partir da busca nas bases de dados da National Library of Medicine (PubMed/

\footnotetext{
${ }^{3}$ Problemas Relacionados a Medicamentos (PRM) são entendidos como os resultados clínicos negativos derivados da farmacoterapia, tidos durante o processo de prescrição ou monitorização dessa farmacoterapia - esses problemas impedem o alcance dos objetivos terapêuticos pretendidos ou colaboram para o surgimento de (novos) efeitos não desejados.
} 
MEDLINE), Scientific Electronic Library Online (Scielo), Biblioteca Virtual em Saúde (BVS), Literatura Latino-americana e do Caribe em Ciências da saúde (LILACS) e Google Scholar. Não houve um corte no tempo das publicações, por entender-se a existência de publicações relevantes para a temática em estudo a qualquer tempo; além de que se buscou materiais a partir dos Descritores em Ciências da Saúde (DeCS/MeSH) em português: Idosos; Prescrições; Uso de Medicamentos; Prescrição Inadequada; Indicadores de Qualidade em Assistência à saúde; Polimedicação; Interações Medicamentosas; Reações Adversas Relacionados a Medicamentos; Estratégias; e em inglês: Aged; Prescriptions; Drug Utilization; Inappropriate Prescribing; Quality indicators in Health Care; Polypharmacy; Drug Interactions; Adverse Reactions; Strategies; utilizando os operadores booleanos "or" e "and" para conectar os termos entre si, abrangendo os resultados de pesquisa para os termos oficiais e seus sinônimos, tornando-a mais específica.

Como critérios de inclusão, foram considerados artigos originais em inglês e português, sem um período determinado, e foram excluídos os artigos duplicados e os que não abordassem o objetivo do estudo. A seleção dos estudos foi realizada em duas etapas. Na primeira etapa, houve a leitura superficial - título e resumo; e na segunda etapa realizou a leitura na íntegra dos artigos previamente selecionados, totalizando 109 artigos que cumpriram os critérios de elegibilidade e se comprovaram úteis para contextualizar e/ou evidenciar com o tema dessa revisão narrativa.

Diante disso, o estudo em sua base metodológica visa colaborar com a educação continuada de profissionais de saúde acerca da importância da utilização de indicadores da prescrição, seus instrumentos e medidas, para avaliação da prescrição em idosos no sistema de saúde, a fim de proporcionar o uso racional e seguro da terapêutica medicamentosa com vistas a uma melhor qualidade de vida ao idoso, e promover um envelhecimento saudável.

\section{Resultados e Discussão}

A busca inicial nas bases de dados identificou 236 artigos, que após a primeira e segunda etapa de seleção resultou em uma amostra de 109 artigos elegíveis; em que o estudo mais antigo encontrado foi Beers et al. (1991) e o estudo mais atual foi Mercadante et al. (2021). Discorreremos essa revisão narrativa sobre esses artigos selecionados em três tópicos: o primeiro tópico, demonstrando a relação do uso racional de medicamentos com a qualidade da prescrição; segundo tópico abordando a avaliação da qualidade da prescrição; e o terceiro tópico descrevendo os indicadores de qualidade da prescrição, seus instrumentos e medidas como estratégias para serem usados no sistema de saúde.

\subsection{Uso racional de medicamentos/ Qualidade da prescrição}

$\mathrm{O}$ uso não racional de medicamentos tem se tornado um problema de ordem mundial $-50 \%$ dos medicamentos são prescritos, dispensados, vendidos ou utilizados inapropriadamente; e é ainda mais desafiador dentro do cenário dos países em desenvolvimento, que apresentam uma população idosa sem assistência integral à saúde. Segundo a Organização Mundial da Saúde (OMS), a definição do Uso racional de medicamentos (URM) é quando os pacientes recebem medicamentos apropriados (adequados) às suas necessidades clínicas, seguros e eficazes, em doses e intervalos ajustados às suas particularidades individuais, por período de tempo necessário, com acesso e baixo custo, assegurando a saúde (World Health Organization, 1987; World Health Organization, 2002; Franceschet-de-Sousa, et al., 2010).

A prescrição de medicamentos para idosos têm evidenciado fatores como a formação deficiente do profissional da saúde sobre o assunto, falta de cuidado e visão integral da saúde do idoso, polifarmácia, o uso de medicamentos inapropriados, gerando IM, RAM e cascata iatrogênica, problemas de não adesão ao tratamento medicamentoso, levando o idoso a eventos adversos como: declínio funcional e cognitivo, quedas e hospitalizações, que na maioria das vezes são evitáveis (Pereira, et al., 2004; Castellar et al., 2007; Baldoni et al., 2010; Shah \& Hajjar, 2012; Lu et al., 2015; Mercadante et al., 2021). Em um estudo 
de coorte norte-americano foram descobertos eventos adversos graves, que ameaçam a vida, e poderiam ter sido impedidos no momento da prescrição, durante o acompanhamento do paciente ou na fase de adesão ao tratamento. Sabe-se que uma prescrição inadequada, faz com que ocorra o uso impróprio de medicamentos, consequentemente implicações como: fármacodependência, efeitos adversos, toxicidade, eficácia limitada, resistência a antimicrobianos, entre outros (Gurwitz et al., 2003; Pereira, et al., 2004; Franceschet-de-Sousa, et al., 2010; Reich, et al., 2014; Silva, et al. 2018).

A qualidade de uma prescrição é compreendida como o equilíbrio entre conhecimento científico, as necessidades individuais do paciente e questões econômicas e familiares - tornando-a uma "prescrição adequada" ou "apropriada". Seu inverso, uma "prescrição inadequada" ou "inapropriada" é o uso de um medicamento que representa mais risco que benefício ao paciente (Galvão \& Ferreira, 2006; Spinewine, et al., 2007; Steinman \& Hanlon, 2010; Scott \& Jayathissa, 2010).

Ainda, a inadequação inclui a omissão de um medicamento necessário (underprescribing); a prescrição de um medicamento desnecessário (overprescribing); o uso de diferentes medicamentos concomitantes (polifarmácia); prescrição incorreta de um medicamento necessário (misprescribing) - que se refere a dosagens erradas, duplicidade terapêutica, medicamentos que possam provocar interações medicamentosas graves, reações adversas e o uso de medicamentos que devem ser evitados, ou seja, contraindicados, ou sem eficácia comprovada (Spinewine, et al., 2007; Scott \& Jayathissa, 2010). Pesquisadores apontam que entre $14 \%$ e $20 \%$ dos idosos são vítimas de prescrição excessiva ou incorreta, recebendo ao menos um medicamento inapropriado - onde os riscos potenciais superam os benefícios (Costa, et al., 2008; Costa \& Pedroso, 2011; Sousa-Muñoz, 2012; Freitas \& Reis, 2018;).

Para a promoção do URM, tem-se utilizado um enfoque mais didático, sendo considerada uma estratégia mundial: pela preferência de medicamentos essenciais ${ }^{4}$ e o estabelecimento de condutas pautadas pelas melhores evidências disponíveis. Dessa forma, é proposto verificar os indicadores da prescrição, seus instrumentos e medidas, como: considerações, reflexões, critérios, escalas e protocolos, guiando profissionais de saúde a introduzir estratégias e práticas com a finalidade de se obter efetividade e segurança, sendo de real relevância, uma vez que os idosos são mais propensos a eventos adversos, maior número de medicamentos, consultas, hospitalizações e gastos com a saúde (Avorn, 2002; Pereira et al., 2004; Ribeiro at al., 2005; Wannmacher, 2006; Spinewine et al., 2007; Steinman \& Hanlon, 2010; Baldoni et al., 2010; Castilho et al., 2018, Silva et al., 2018).

\subsection{Avaliação da Qualidade da prescrição}

A avaliação da qualidade da prescrição se dá pela verificação dos indicadores com instrumentos e medidas como ferramentas ou estratégias clínicas e educacionais, que visam guiar a utilização de medicamentos nos idosos e são considerados recursos fundamentais para os profissionais que buscam apoio baseado no binômio consenso/evidências (Lucchetti \& Lucchetti, 2017).

Esses instrumentos e medidas de avaliação se baseiam no julgamento clínico, sendo critérios de discernimentos implícitos ou explícitos. Os critérios implícitos são específicos e avaliam a qualidade/adequação da prescrição segundo o julgamento clínico profissional para um paciente individualmente, tendo em conta as práticas mais apropriadas nas revisões de literatura médica. São demorados e dependentes de conhecimentos e atitudes profissionais, demonstrando baixa confiabilidade; os critérios explícitos, são os mais utilizados e aceitos pela comunidade científica e envolvem a opinião de especialistas e

\footnotetext{
${ }^{4}$ Medicamentos essenciais são aqueles que satisfazem as necessidades prioritárias de cuidados da saúde da população, e que devem ser selecionados por critérios de eficácia, segurança, conveniência, qualidade, comparação de custo favorável, e estarem disponíveis em todos os momentos, dentro do contexto de funcionamento dos sistemas de saúde, em quantidades adequadas e dosagem apropriada.
} 
técnicas de consenso, como o método Delphi ${ }^{5}$ na sua elaboração - na maioria das vezes esses métodos são orientados pelo medicamento ou condição clínica, podendo ser aplicados com pouca ou nenhuma avaliação clínica, utilizando somente dados da prescrição ou associados aos dados clínicos, sendo inclusive proveitosos na avaliação de grandes bancos de dados (Willcox, et al., 1994; Galvão \& Ferreira, 2006; Spinewine et al., 2007; Scott \& Jayathissa, 2010; Costa \& Pedroso, 2011; Freitas \& Reis, 2018; Lucchetti, \& Lucchetti, 2017; Marques \& Freitas, 2018; Praxedes, et al., 2021).

Esses critérios explícitos são de alta confiabilidade e reprodutibilidade, contudo, apresentam como desvantagem a possibilidade de se produzir resultados incorretos, por não considerarem todas as características e/ou necessidades individuais. Porém, estudos evidenciaram que as utilizações desses critérios resultam em menor IM potencial e menor risco de RAM graves (Spinewine, 2007; Scott \& Jayathissa, 2010; Dimitrow et al., 2011; Freitas \& Reis, 2018; Marques \& Freitas, 2018; Praxedes, et al., 2021).

As medidas de avaliação para investigar a qualidade da prescrição, o uso de medicamentos ou auxiliar nas intervenções são úteis para otimizar uma atenção apropriada à população idosa. Assim, o uso dessas medidas/instrumentos auxiliam o profissional de saúde a questionar a escolha, se um medicamento foi utilizado ou não, de acordo com os padrões farmacológicos aceitos, ou auxiliam na percepção do resultado - se um medicamento conduziu a uma RAM, admissão hospitalar ou outra consequência indesejável (Pereira, et al., 2004; Ribeiro, et al., 2005; Spinewine, et al., 2007; Scott \& Jayathissa, 2010; Gallagher, et al., 2008; Costa, et a., 2008; Portela, et al., 2010; Steinman \& Hanlon, 2010; Freitas \& Reis, 2018; Sousa-Muñoz, et al., 2012; Kaufmann, et al., 2012; Lucchetti \& Lucchetti, 2017).

\subsection{Indicadores de qualidade da Prescrição: Instrumentos e medidas como estratégias}

\subsubsection{Polifarmácia, reações adversas, interações medicamentosas}

A grande maioria dos idosos consomem medicamentos com mais frequência e de forma mais intensa que o restante da população, e a prevalência da polifarmácia - definida como o uso regular de 5 ou mais medicamentos - é maior nessa população, associada ao aumento na prevalência de multimorbidade crônica e as mudanças funcionais que ocorrem com o envelhecimento (Rozenfeld, 2003; Carvalho, et al., 2012; Baldoni et al., 2013; Ramos et al., 2016; Mercadante, et al., 2021).

Pesquisadores apontam que de todos os medicamentos prescritos para a população brasileira, 50\% são utilizados por idosos, sendo que um número crescente de prescrições acompanha o envelhecimento populacional. A prevalência de polifarmácia pode variar de acordo com a população estudada. Um estudo na Escócia estimou uma prevalência de $21 \%$ em idosos da comunidade; outro na Coreia do Sul demonstrou uma prevalência de $86 \%$ em idosos que visitaram clínicas e hospitais; no Brasil, alguns estudos populacionais reportaram a prática da polifarmácia entre os idosos da comunidade: $50 \%$ no Estudo Epidoso, 36\% no Estudo SABE, - ambos em São Paulo - e 32\% no Estudo EpiFloripa em Florianópolis, bem como, dados da Pesquisa Nacional de Acesso, Utilização e Promoção do Uso Racional de Medicamentos apontam que a polifarmácia foi maior entre os mais idosos (+80) 20,0\% (Penteado, et al., 2002; Costa, et al., 2008; Carvalho, et al., 2012; Kim et al., 2014; Guthrie et al., 2015; Ramos, et al., 2016; Pereira, et al., 2017; Mercadante, et al., 2021).

A polifarmácia está relacionada com diferentes fatores como idade, multimorbidade, principalmente as doenças crônicas cardiovasculares, automedicação, cascata iatrogênica, elevado consumo de medicamentos isentos de prescrição médica, baixa escolaridade, sexo feminino, uso de medicamento sem indicação clara, maior procura pelos serviços de saúde, além do alto consumo de terapias alternativas e principalmente prescrição inadequada, seja pela subutilização de fármacos necessários e/ou o uso inadequado de especialidades terapêuticas. Ratifica-se: a terapêutica é inadequada quando impõe riscos

\footnotetext{
${ }^{5}$ Método Delphi é uma técnica de investigação que permite reunir um conjunto de opiniões de especialistas, separados geograficamente, levando a resultados densos sobre temáticas complexas e abrangentes.
} 
Research, Society and Development, v. 11, n. 2, e55311225942, 2022

(CC BY 4.0) | ISSN 2525-3409 | DOI: http://dx.doi.org/10.33448/rsd-v11i2.25942

excessivos comparados com os seus benefícios potenciais (Fulton \& Allen, 2005; Janius-Walker, et al., 2007; Lai, et al., 2010; Carvalho et al., 2012; Lu, et al., 2015; Fustinoni, et al., 2015; Mercadante, et al., 2021).

A prática da polifarmácia, comumente oriunda de prescrição e uso inadequado ou excessivo de medicamentos, é considerada um indicador de qualidade da prescrição, pois pode apresentar várias implicações como: maior risco de IM e RAM, a não adesão à terapia medicamentosa, desenvolvimento de síndromes geriátricas, redução da capacidade funcional e cognitiva, maiores custos com saúde e hospitalizações, podendo intensificar doenças pré-existentes, comprometer o estado geral de saúde do idoso e aumentar a necessidade de cuidados especiais e maiores investimentos destinados à saúde (Penteado et al., 2002; Castellar, et al., 2007; Lai et al., 2010; Shah \& Hajjar, 2012; Lu et al., 2015; Mercadante et al., 2021).

Em relação a prática da polifarmácia nos idosos favorecer uma não adesão terapêutica, pode ser devido a esquemas terapêuticos complexos, pela quantidade de medicamentos e consequentemente aumento no número de tomadas, onde o paciente pode não cumprir a prescrição - por falta de compreensão/informação ou de forma intencional - ocorrendo a progressão da doença ou falha do tratamento. As taxas de adesão entre idosos residentes em comunidade variam entre $43 \%$ e 95\% (Diaz, 2002; Rocha, et al., 2008; Arruda, et al., 2015).

Além da polifarmácia, a investigação de ocorrência de RAM também é considerada um indicador de qualidade da prescrição, pois as RAM representam a quinta maior causa de morte, e são responsáveis por cerca de 3\% a 6\% das internações hospitalares nas diferentes faixas etárias, podendo atingir 24\% dos idosos, além de representar 5\% a $10 \%$ dos gastos hospitalares. Decorrem de alterações farmacocinéticas e farmacodinâmicas relacionadas ao envelhecimento, prescrição de polifarmácia feita por mais de um médico, uso incorreto de medicamentos, e prescrição mais frequente de fármacos com margem de segurança terapêutica reduzida (Onder et al., 2002; Passarelli et al., 2005; Castellar, et al., 2007; Secoli, 2010; Scott \& Jayathissa, 2010).

O estudo de Gurwitz et al. (2003) demonstrou que 0,7\% dos eventos adversos em idosos são fatais, 8,9\% ameaçam a vida, $28,3 \%$ são eventos sérios e $62 \%$ são significantes, sendo que 27,6\% são considerados eventos adversos evitáveis, provenientes da prescrição inadequada de medicamentos entre os idosos. O risco de RAM com o uso concomitante de dois fármacos é de $13 \%$, valor que chega a $58 \%$ quando se administram cinco medicamentos, e alcança $82 \%$ quando a farmacoterapia chega a sete ou mais (Secoli, 2010).

Assim, a probabilidade de uma RAM deve ser sempre ponderada durante a avaliação de um idoso: qualquer novo sintoma deve ser relacionado ao medicamento, como um efeito adverso desse medicamento, e não como uma nova condição médica que exige nova prescrição - cascata iatrogênica - expondo o paciente a desenvolver risco de efeitos prejudiciais adicionais relacionados ao tratamento potencialmente desnecessário. Vale ressaltar que, quanto maior o número de fármacos prescritos em conjunto (polifarmácia), maior a chance da ocorrência de interação medicamentosa (Rochon \& Gurwtz, 1995; Bjorkman, et al., 2002; Scott \& Jayathissa, 2010; Rochon, et al., 2021).

Outro indicador importante para se investigar na avaliação da qualidade da prescrição são as interações medicamentosas, muito frequentes entre os idosos. Sua gravidade, importância, prevalência e possíveis consequências estão, principalmente, relacionadas a prática da polifarmácia, onde há possibilidade de ocorrer influência de um medicamento na ação de outro; assim como, também, estão relacionadas com as variáveis: genética, idade, multimorbidades, situação das funções renal e hepática, consumo de álcool, tabagismo, fatores ambientais, características dos medicamentos, fragilidade, estado nutricional e alterações farmacocinéticas e/ou farmacodinâmicas dos medicamentos no envelhecimento, que podem levar a soma de efeitos adversos quando, principalmente, os fármacos associados têm o mesmo perfil toxicológico (Bjorkman, et al., 2002; Locatelli, 2007; Mallet, et al., 2007; Correr et al., 2007; Costa \& Pedroso, 2011; Correr, Otuki, 2013)

Prescrições têm apresentado ao menos uma combinação de medicamentos com potencial interação, podendo ser evitáveis, bem como o número de medicamentos está associado a um maior risco de interações medicamentosas. As interações 
Research, Society and Development, v. 11, n. 2, e55311225942, 2022

(CC BY 4.0) | ISSN 2525-3409 | DOI: http://dx.doi.org/10.33448/rsd-v11i2.25942

medicamentosas revelam o uso inadequado de medicamentos, pois são capazes de causar lesões permanentes, algumas provocando efeitos restritivos no paciente: necessidade de hospitalizações, aumento no tempo de internação, na duração do tratamento ou na ação de determinado medicamento; seja porque ocorre aumento ou redução da eficácia de um ou de ambos medicamentos, ou o aparecimento de um novo efeito não observado com o uso isolado de qualquer um dos medicamentos envolvidos (Bjorkman, et al., 2002; Locatelli, 2007; Mallet, et al., 2007; Costa \& Pedroso, 2011; Kaufmann et al., 2014).

Assim, uma das estratégias de intervenção para promover uma prescrição de qualidade é reduzir a polifarmácia e, consequentemente, seus riscos associados como a IM e as RAM, ou seja, a desprescrição - considerada um critério implícito, sua prática consiste no processo de identificar e descontinuar medicamentos desnecessários, inefetivos, inseguros ou potencialmente inapropriados, e na cooperação entre profissionais de saúde e pacientes, para que possa gerir e monitorar a farmacoterapia (Doron, et al., 2015; McGrath, et al., 2017; Institute for Safe Medication Practices Canada (2018); Rochon, et al., 2021).

Segundo McGrath et al. (2017) e Institute for Safe Medication Practices Canada (2018), a desprescrição deve considerar os benefícios e prejuízos do medicamento ao paciente, a finalidade do tratamento medicamentoso, esperança de vida do paciente, bem como a comodidade e as escolhas do paciente que possam contribuir para a adesão ao tratamento minimizando riscos. O conhecimento do paciente sobre desprescrição deve ser estimulado, como a de todos os profissionais que cuidam do paciente, a participação do paciente e de seus familiares de forma engajada em seu cuidado é o mais importante para a qualidade e segurança dele (Correr \& Otuki, 2013; Doron, et al., 2015; Mcgrath, et al., 2017; Institute for Safe Medication Practices Canada, 2018; Rochon, et al., 2021).

Contudo, é preciso que haja um equilíbrio entre o excesso e a omissão de medicamentos, atentando que muitas vezes se faz preciso prevalecer uma diversidade de medicamentos, para gerir a farmacoterapia nos idosos com multimorbidade que, nesse caso, é indicado o uso; todavia um enfoque mais metódico é necessário para orientar os regimes terapêuticos às necessidades deles, ou um julgamento cuidadoso dos medicamentos para averiguar se devem ser descontinuados ou substituídos (Penteado, et al., 2002; Correr, et al., 2011; Correr \& Otuki, 2013; Mercadante, et al., 2021). Outra estratégia de intervenção, relacionada a polifarmácia, é a conciliação de medicamentos, também considerada um critério implícito. A conciliação consiste em avaliar se todos os medicamentos em uso pelo paciente estão coerentes com as prescrições médicas, se há duplicidades terapêuticas ou abandono de tratamentos que deveriam ser utilizados (Correr, et al., 2011; Correr \& Otuki, 2013; Lira \& de Oliveira, 2021).

Em relação ao indicador da prescrição "IM", pode-se se valer de estratégias com o uso de ferramentas, como banco de dados do UpToDate (Lexicomp®) Drug Interaction Checker (Medscape), University of Maryland Medical Center Drug Checker (Micromedex) e o Sitio drugs.com, consideradas critérios explícitos, para investigar as IM e sua gravidade. Essas bases de dados proporcionam informações de segurança para prevenção e/ou diminuição de eventos adversos, já que as interações podem afetar a efetividade e segurança de um regime terapêutico influenciando na alteração das doses, ou ainda, no início do uso de um novo medicamento e a promoção das melhores práticas clínicas para o acompanhamento de pacientes. Como estratégia utilizada para a averiguação do indicador da prescrição "RAM" é necessário, primeiramente, que ocorra a notificação da reação adversa para que se possa investigar a causalidade por meio de ferramentas como os algoritmos (critérios explícitos) - onde mais utilizados na prática clínica são: Invima, Karch e Lasagna, e Naranjo; para estabelecer uma relação da reação e o medicamento sob suspeita de ocasionar a mesma, definindo o grau de imputabilidade: definida/certa, provável/possível/improvável (Côrrea, et al., 2017; Yamagata, et al., 2018).

Desse modo, essas estratégias auxiliam a promover e avaliar a qualidade da prescrição e uma correta utilização dos medicamentos, que evita riscos e resultados negativos, como a diminuição do status funcional e cognitivo, redução da qualidade de vida, hospitalização, custos em saúde ou mesmo, o óbito (Hepler \& Strand, 1990; Rovers \& Currie, 2010; Correr, 
et al., 2011; Correr \& Otuki, 2013).

\subsubsection{Medicamentos Inapropriados e critérios como estratégias para promover qualidade da prescrição}

Os medicamentos potencialmente inapropriados para idosos (MPII) são assim considerados por falta de evidências ou não comprovação de eficácia - onde o alto risco de eventos adversos superam os benefícios. $\mathrm{O}$ uso desses fármacos não é proibido, porém o ideal é que sejam evitados em idosos, independentemente da dose e do tratamento utilizados. Contudo, apesar das evidências de maus resultados, os MPII continuam sendo prescritos e usados como tratamento de primeira linha para a maioria dos idosos (Gallagher, et al., 2008; Gorzoni, et al., 2012; AGS, 2015).

Em diversos países a prevalência de MPII em idosos é elevada, variando, de acordo com a ferramenta de triagem utilizada, entre $33,9 \%$ e $58 \%$ no contexto domiciliar e entre $42,4 \%$ e $60,5 \%$ em idosos hospitalizados. No Brasil, a prevalência chega a 59,2\% em idosos residentes na comunidade e a $85,9 \%$ em idosos internados. O uso está associado com o sexo feminino, idade avançada e com o número de drogas prescritas (polifarmácia), depressão, insuficiência cardíaca e incapacidade funcional; e, a presença, na prescrição, de ao menos um medicamento inapropriado, dobra o risco de desencadeamento de RAM. Os MPII têm alta prevalência também na Europa e nos EUA, e dentre as especialidades médicas que mais prescrevem os medicamentos considerados inadequados, sobressai a cardiologia (48\%), contrapondo a geriatria que apresenta uma menor taxa (24\%) dentre as prescrições inadequadas (Gallagher, et al., 2008; Rothberg, et al., 2008; Guaraldo, et al., 2011; Praxedes, et al., 2021).

Existem diversos critérios - instrumentos/medidas de avaliação - como estratégia para qualidade da prescrição, desenvolvidos para verificar se o medicamento é apropriado ou não, porém, os critérios explícitos mais utilizados mundialmente são os Critérios de Beers e o STOPP-START, pois proporcionam maior capacidade de detectar e prevenir as IM e RAM ocasionados por esses medicamentos. $\mathrm{O}$ uso desses critérios tende a aprimorar a prescrição medicamentosa nos idosos, garantindo qualidade e segurança. No entanto, nenhum critério que avalia se os medicamentos são inapropriados deve ser empregado como uma lista proibitiva de medicamentos, mas deve ser aproveitado de forma que auxilie o monitoramento de eventos adversos e iatrogênias, e forneça uma terapia medicamentosa mais segura (Oliveira, et al., 2016; Freitas \& Reis, 2018; Praxedes, et al., 2021).

O critério STOPP/START (Screening Tool of Older Persons Prescriptions/Screening Tool to Alert to Right Treatment) foi desenvolvido em 2008 por meio do método Delphi. A atualização de 2015, conta com 81 critérios do STOPP, clinicamente significativos para a prescrição potencialmente inapropriada, e 34 critérios do START baseados em evidências para omissões na prescrição, ambos organizados por sistema fisiológico, onde cada medicamento é seguido de uma breve explanação sobre a inadequação do seu uso. Esse critério avalia ainda, a interação medicamento-medicamento, medicamentodoença, a duplicidade terapêutica, os fármacos que aumentam o risco de deterioração cognitiva e os fármacos que aumentam o risco de quedas (Willcox et al., 1994; O’mahony et al., 2015; Freitas \& Reis, 2018; Marques \& Freitas, 2018).

Em relação aos Critérios de Beers, sua concepção também se deu como uma opção eficaz de investigar e avaliar a qualidade da prescrição, de forma a estudar e prevenir o uso de medicamentos inadequados para os idosos com enfoque nas evidências, baseando-se no método Delphi. Os Critérios de Beers foram revisados, expandidos e atualizados em 1997, 2003, 2012, 2015 e 2019 para incluírem todos os setores de atendimento geriátrico, e foram divididos em dois grupos: o primeiro grupo de medicamentos ou classes que devem ser evitados em idosos, independentemente do diagnóstico ou da condição clínica, devido ao alto risco de eventos adversos e pela existência de outros fármacos mais seguros; e o segundo grupo, de medicamentos ou classes que não devem ser utilizados em determinadas circunstâncias clínicas. Com as atualizações, os critérios têm evidenciado eficiência na prevenção de RAM no idoso, na redução das internações hospitalares, diminuição das consultas de emergência, e consequentemente, no barateamento dos gastos para o sistema de saúde e usuários (Beers, et al., 
1991, AGS, 2015; AGS, 2019; Oliveira, et al., 2016; Marques \& Freitas, 2018; Freitas \& Reis, 2018; Praxedes, et al., 2021).

Esses critérios apresentaram diferenças para algumas realidades, reforçando a relevância de se desenvolver critérios nacionais para detectar MPII. O Consenso Brasileiro de Medicamentos Potencialmente Inapropriados para Idosos, o primeiro realizado no Brasil, orienta profissionais de saúde no cuidado do idoso, buscando aplicar uma lista de MPII adequada à realidade brasileira. Assim, foi desenvolvida uma lista de 43 critérios de medicamentos que devem ser evitados em idosos, independentemente da condição clínica, e 75 critérios que dependem da condição clínica do paciente (Oliveira, et al., 2016).

Estudos têm apontado uma forte relação entre os MPII e desfechos desfavoráveis em idosos, como: interações medicamentosas, RAM, hospitalizações e mortalidade. Além do mais, há evidências de que vários MPII estão associados a problemas graves, como: delirium, déficit cognitivo, hemorragia gastrointestinal, quedas e fraturas (Lu et al., 2015; Praxedes, et al., 2021)

Expor o idoso a interações medicamentosas e RAM em troca de um beneficio muito restrito de determinado medicamento, é arriscado. Portanto, vale ressaltar a importância de revisar a farmacoterapia dos idosos, gerenciando e monitorando-a rigorosamente por meio do indicador "MPII" e medidas de avaliação da prescrição, como esses critérios, para elevar ao máximo o benefício farmacológico, reforçando a adesão ao tratamento, minimizando riscos, reduzindo gastos e prevenindo complicações (Passarelli, et al., 2005; Gallagher, et al., 2008; Gorzoni, et al., 2012; Mcgrath, et al., 2017; Freitas \& Reis, 2018; Praxedes, et al., 2021).

\subsubsection{Medicamentos Anticolinérgicos, carga anticolinérgica e escalas como estratégias para promover qualidade da prescrição.}

Os medicamentos anticolinérgicos também servem como um indicador de qualidade da prescrição. O termo utilizado “medicamento anticolinérgico" é empregado não somente para os medicamentos desenvolvidos por sua atividade anticolinérgica, mas também para todo medicamento que é capaz de produzir efeitos anticolinérgicos, como por exemplo: os antidepressivos, antipsicóticos, relaxantes musculares, fármacos que atuam no sistema cardiovascular, fármacos que atuam ao nível gastrointestinal, antiespasmódico, anti-histamínicos, antiepiléticos, benzodiazepínicos, broncodilatadores, diuréticos, antiparkinsonianos e opióides. Esses medicamentos se ligam competitivamente aos receptores nicotínicos e muscarínicos, inibindo as respostas mediadas pela acetilcolina ao nível dos órgãos efetores (Rang, et al., 2012; Persaud, et al., 2014; Brunton, et al., 2018).

A acetilcolina faz diversas ações periféricas e centrais, mas principalmente tem um papel fundamental na plasticidade sináptica necessária à aprendizagem e memória, em que os neurônios colinérgicos exercem influência sobre a cognição, atenção, sono, metabolismo e modulação de outros neurotransmissores. Já os medicamentos anticolinérgicos ou "antagonistas colinérgicos" agem inibindo essas ações, ocasionando principalmente ao nível do Sistema Nervoso Central (SNC), agitação, desorientação, sedação, incluindo efeitos antieméticos e efeitos antiparkinsonianos, através da ação sobre o sistema extrapiramidal (Gerretsen \& Pollock, 2013; Rang, et al., 2012; Persaud, et al., 2014; Brunton, et al., 2018).

Os efeitos adversos oriundos do uso de medicamentos anticolinérgicos podem ser centrais, tais como: comprometimento cognitivo, déficit de memória, confusão, sintomas psicóticos, delirium, tonturas, excitação comportamental, déficits de atenção, alucinações e alterações no equilíbrio, podendo contribuir para quedas e acidentes. Além, podem ser percebidos efeitos periféricos como: obstipação, redução do peristaltismo, xerostomia, diminuição do esvaziamento gástrico, diminuição da acidez gástrica, retenção urinária, secura ocular, visão turva, midríase e taquicardia (Persaud et al., 2014; Ruxton et al., 2015; Salahudeen et al., 2015). Esses efeitos podem incidir tanto em doses tóxicas como terapêuticas, quando utilizados em pessoas vulneráveis como idosos ou portadores de doenças mentais. Contudo, é necessário relembrar que no envelhecimento há alterações farmacodinâmicas e farmacocinéticas, o que predispõe os idosos aos efeitos adversos dos 
medicamentos anticolinérgicos. Entretanto, muitos desses efeitos podem ser negligenciados e perigosamente considerados parte do processo de envelhecimento ou da progressão de uma doença subjacente (Gerretsen \& Pollock, 2013; Rang et al., 2012; Cardwell et al., 2015; Salahudeen et al., 2015; Brunton, et al., 2018).

Cerca de $20 \%$ a $50 \%$ das pessoas idosas receberam prescrição de ao menos um medicamento com atividade anticolinérgica (Campbel, et al., 2009). Estudos de revisão sistemática que incluíram dados de ensaios clínicos e estudos observacionais evidenciou uma redução significativa da capacidade cognitiva, aumento da multimorbidade e mortalidade relacionada com o tratamento anticolinérgico (Fox et al., 2014; Salahudeen, et al., 2015). Além dos medicamentos anticolinérgicos estarem associados ao comprometimento da função cognitiva, eles também estão associados à perda de independência, à diminuição da mobilidade, ao elevado risco de quedas, hospitalizações, institucionalizações e mortalidade por todas as causas (Fox, et al., 2014; Salahudeen, et al., 2015; Myint et al., 2015).

Entretanto, deve-se ter atenção, principalmente, com a administração de anticolinérgico em pacientes com Alzheimer em uso de anticolinesterásicos ${ }^{6}$ - pois esses pacientes apresentam alto risco de deterioração cognitiva secundária a medicamentos com efeitos anticolinérgicos, uma vez que, já exibem uma marcada redução no funcionamento das vias centrais colinérgicas, fundamental para o funcionamento dos processos mnésicos. Um estudo apontou o uso de anticolinérgico em pacientes com Alzheimer concomitante a anticolinesterásico, e consequente houve queda no escore do Mini-Mental State Examination (MMSE) em relação a pacientes com Alzheimer sem prescrição simultânea de anticolinérgico (Lu \& Tune, 2003; Hori, et al., 2014; Cardwell et al., 2015; Salahudeen et al., 2015; Brunton, et al., 2018).

Os medicamentos anticolinérgicos são frequentemente mencionados nas listas de MPII, como por exemplo, nos Critérios de Beers, e a partir da atualização de 2015 é que se foi recomendado evitar e/ou minimizar o uso concomitante de diversos fármacos anticolinérgicos devido a diferentes efeitos adversos, principalmente, os efeitos a nível do SNC (Baldoni, et al., 2014; AGS, 2015; AGS, 2019). Contudo, tem se percebido que muitos idosos utilizam diversos medicamentos com efeito anticolinérgico para várias condições crônicas, o que produz uma exposição cumulativa, descrita na literatura como Carga Anticolinérgica (CAC). Os efeitos adversos dos anticolinérgicos nem sempre estão associados diretamente com o uso de um único medicamento com fortes propriedades anticolinérgicas, mas podem refletir o acúmulo de múltiplos fármacos com vários graus de efeitos anticolinérgicos (Rudolph, et al., 2008; Ruxton, et al., 2015; Persaud, et al., 2014; Salahudeen, et al., 2015; Borja-Oliveira, 2017).

A variabilidade farmacocinética e farmacodinâmica individual, número de medicamentos prescritos (polifarmácia), automedicação, dosagens, interações medicamentosas, e a prevalência e gravidade das multimorbidades, também podem influenciar a CAC cumulativa e a gravidade dos eventos adversos em idosos (Gerretsen \& Pollock, 2011; Pasina, et al., 2013; Lu et al., 2015; Wan-Hsuan, et al., 2015).

Diversos estudos têm correlacionado a CAC com o déficit cognitivo e funcional, bem como risco elevado de quedas, hospitalizações, institucionalizações, baixa qualidade de vida, morbimortalidade em idosos, sendo tal relação mais forte com o aumento da carga anticolinérgica, bem como uso crônico de anticolinérgico que pode também elevar o risco de demência e incapacidade funcional (Gerretsen \& Pollock, 2013; Fox, et al., 2014; Ruxton, et al., 2015; Salahudeen, et al., 2015). O estudo de Wehling (2009) conduzido em idosos, residentes na comunidade, constatou que a alta carga de medicamentos anticolinérgicos esteve associada com uma pior performance relativa a mobilidade e execução de testes cognitivos; entretanto, no estudo de Hilmer et al (2009) é evidenciado que quando os medicamentos anticolinérgicos foram interrompidos houve diminuição do risco de comprometimento cognitivo e funcional.

Diante disso, a análise da CAC tem sido utilizada como indicador de qualidade na prescrição de idosos, e estratégias

${ }^{6}$ Medicamento anticolinesterásico: inibem as colinesterases, potencializando a ação da acetilcolina nas sinapses colinérgicas. 
Research, Society and Development, v. 11, n. 2, e55311225942, 2022

(CC BY 4.0) | ISSN 2525-3409 | DOI: http://dx.doi.org/10.33448/rsd-v11i2.25942

são imprescindíveis para diminui-la, a fim de trazer benefícios significativos para a saúde (Shekelle et al., 2001; Cardwell, et al., 2015; Lu, et al., 2015; Ruxton, et al., 2015; Salahudeen, et al., 2015). Atualmente, pode-se verificar a atividade anticolinérgica de um fármaco por meio da medida da atividade anticolinérgica sérica (SAA) - seus metabólitos e fisiologia do paciente, afinidade para receptores muscarínicos in vitro - porém, essa medida é dispendiosa e indisponível para muitos prescritores, um fator impeditivo na prática clínica (Lertxundi, et al., 2013; Hori, et al., 2014). Entretanto, também é possível verificar a atividade anticolinérgica por meio de escalas - medidas de avaliação da qualidade da prescrição (critérios explícitos) - combinada ao julgamento clínico, com a finalidade de mensurar a CAC dos medicamentos, a potencialidade de produção de efeitos adversos e o risco à exposição para esses fármacos. Apesar de existir essa diversidade de medidas de avaliação, não existe uma prática padrão para medir a exposição aos anticolinérgicos (Gerretsen \& Pollock, 2013; Salahudeen, et al., 2015; Mayer, et al., 2015; Hsu, et al., 2017).

Essas escalas foram desenvolvidas como estratégias para assessorar profissionais de saúde na prática clínica e pesquisa, com o objetivo de orientar no processo de prescrição, de modo a proporcionar qualidade e facilidade do regime terapêutico, otimizando a segurança na farmacoterapia do idoso (Rudd, et al., 2005; Land, et al., 2014; Hori, et al., 2014; Mayer, et al., 2015).

Ao longo dos anos, têm-se desenvolvido diferentes escalas que demonstram a atividade anticolinérgica dos medicamentos e que estimam o risco da exposição a medicamentos com propriedades anticolinérgicas, organizadas de acordo com os saberes de especialistas e conhecimentos disponíveis na literatura (Hsu, et al., 2017; Mayer, et al., 2015; Salahudeen, et al., 2015). Considerado um método simples, rápido e de fácil emprego clínico, entretanto, alguns autores recomendam ainda o emprego de escalas de avaliação cognitiva e funcional para avaliar os efeitos dos anticolinérgicos, já que essas escalas anticolinérgicas podem demonstrar um viés de subjetividade, e a necessidade de certo conhecimento e experiência pelo avaliador em relação aos efeitos negativos dos anticolinérgicos (Durán et al., 2013; Kashyap, et al., 2014; Borja-Oliveira, 2017).

Estudos de revisões sistemáticas demonstraram o uso de três principais escalas na avaliação da CAC: Anticholinergic Drug Scale (ADS); Anticholinergic Risk Scale (ARS); Anticholinergic Cognitive Burden Scale (ACB). Nessas escalas, os medicamentos são ordenados em quatro categorias, variando de "nenhuma atividade anticolinérgica conhecida" $(=0)$, até "alta afinidade anticolinérgica" (=3); e os escores imputados para cada fármaco são somados para definir a pontuação individual do paciente, compondo a carga anticolinérgica. A CAC medida por essas escalas tem sido relacionada com os principais desfechos negativos descobertos pelos estudos na população idosa: déficit cognitivo e funcional (Fox, et al., 2014; Salahudeen, et al., 2015; Ruxton, et al., 2015; Hsu, et al., 2017).

No entanto, estudos apontam que, há reversão do comprometimento funcional e cognitivo quando existe redução da CAC na farmacoterapia do idoso a longo prazo. Desse modo, pesquisadores explicam que se deve evitar admitir que o déficit cognitivo seja demência, até que as presumíveis causas farmacológicas tenham sido rejeitadas (Rudolph, et al., 2008; Durán, et al., 2013; Kashyap, et al., 2014; Ruxton, et al., 2015; Mayer, et al., 2015; Borja-Oliveira, 2017; Hsu, et al., 2017).

A escala ADS, foi desenvolvida por Carnahan et al. (2006), e contém atualmente 117 medicamentos com atividade anticolinérgica, e evidenciou ser útil em diferentes contextos clínicos, bem como empregada para averiguar diversos desfechos adversos como: cognitivos, funcionais, risco de hospitalização e mortalidade (Beuscart, et al., 2014; Ruxton, et al., 2015). O estudo de Beuscart et al. (2014) descreve que 1 a cada 4 idosos institucionalizados foi exposto a uma alta carga anticolinérgica, ou seja, obteve uma prescrição com escore elevado na escala ADS.

A ARS foi desenvolvida por Rudolph et al. (2008), contém 49 medicamentos, e foi traduzida para o português, com ajustes aos medicamentos em uso no Brasil (Rudolph, et al., 2008; Salahudeen, et al., 2015; Gorzoni \& Fabbri, 2017). Um estudo de coorte realizado em idosos hospitalizados com a escala ARS, averiguou-se uma associação entre a exposição da alta 
CAC e o comprometimento cognitivo e funcional. Considerando os desfechos oriundos desse estudo, a escala ARS foi nomeada como melhor ferramenta para identificar idosos com alto risco de comprometimento físico, pois a pontuação elevada da escala ARS evidenciou uma associação negativa com alguns itens do índice de Barthel - escala que verifica as Atividades de Vida Diária (AVDs) (Pasina, et al., 2013). Contudo, o estudo de Gorzoni e Fabbri (2017) no Brasil, analisou 123 prontuários de idosos hospitalizados ao término da internação pela escala ARS e constatou que a pontuação média da ARS foi baixa e não se associou estatisticamente ao efeito adverso delirium, verificado juntamente com o teste Confusion Assessment Method (CAM).

A escala ACB, composta por 88 medicamentos, foi desenvolvida por Boustani et al. (2008), com a intenção de cooperar com a identificação de medicamentos com propriedades anticolinérgicas associados ao comprometimento cognitivo, como: delirium, declínio cognitivo e demência em pessoas idosas, e desse modo, auxiliar na redução de riscos de eventos adversos. Diversos estudos relatam a associação de maior carga anticolinérgica na escala ACB com déficit cognitivo (BorjaOliveira, 2017).

Ainda, é importante nessa revisão, a menção da escala Brazilian Anticholinergic Activity Drug Scale (BADS) ou Escala Brasileira de Medicamentos com Atividade Anticolinérgica, elaborada por Nery e Reis (2019) com a finalidade de avaliar o impacto da carga anticolinérgica nos resultados em saúde, especialmente de idosos, pacientes psiquiátricos e com doença de Parkinson. Foi desenvolvida com uma metodologia simples e sistematizada, possuindo 125 medicamentos que contemplam as especificidades do mercado farmacêutico brasileiro. Contudo, essa escala ainda não foi validada em diferentes cenários assistenciais, assim como, comparada com outras escalas e com desfechos cognitivos e funcionais.

Vale salientar que, até o momento não existe nenhum "padrão ouro" para medir e avaliar a exposição dos idosos aos fármacos com efeitos anticolinérgicos, ou seja, não existe um consenso entre as escalas a respeito dos medicamentos e sua pontuação. Os desfechos podem ser diferentes de acordo com a escala empregada; todavia, essa diferença talvez possa ser explicada pelos diferentes métodos aplicados na elaboração das mesmas, e pela avaliação entre os especialistas, já que são dados que conduzem a variações nos escores atribuídos a cada fármaco de acordo com o seu potencial anticolinérgico (Pont, et al., 2015).

\section{Considerações Finais}

Essa revisão narrativa demonstrou que promover a qualidade da prescrição em idosos é um processo complexo e que envolve diferentes determinantes; contudo, é de suma importância para a promoção do uso racional de medicamentos.

A discussão sobre esse assunto evidencia cada vez mais que essa população é exposta a medicamentos, e esses estão associados ao comprometimento funcional e cognitivo, sendo imperativo realizar a gestão da farmacoterapia na pessoa idosa, uma vez que, os dados apresentados por estudos nessa revisão demonstram que o uso dos indicadores da prescrição, seus instrumentos e medidas, é indispensável no sistema de saúde. Desse modo, para a realização da gestão da farmacoterapia do idoso é necessário estabelecer um balanço entre os efeitos terapêuticos e os eventos adversos, adotando medidas que impactam o ciclo do medicamento no idoso, para que não ocorra um elevado número de hospitalizações, institucionalizações, custos em cuidados de saúde e mortalidade.

Após a reflexão sobre esse assunto, sugere-se que sejam realizados estudos de intervenção, utilizando esses instrumentos e medidas para promover uma prescrição de qualidade para os idosos em diferentes contextos clínicos, provocando a diminuição dos PRMs e dos custos em saúde, com vistas a um serviço de saúde que saiba orientar o idoso e proporcionar um envelhecimento saudável. Esse trabalho e novos que virão sobre esse assunto, podem servir de apoio para educação continuada de profissionais de saúde, bem como, auxiliar na implementação de políticas e estratégias para a gestão 
Research, Society and Development, v. 11, n. 2, e55311225942, 2022

(CC BY 4.0) | ISSN 2525-3409 | DOI: http://dx.doi.org/10.33448/rsd-v11i2.25942

clínica do medicamento nos idosos, já que os fármacos podem interferir na realidade terapêutica dos idosos.

Diante do apresentado, é imprescindível promover uma prescrição de qualidade e adequada, a fim de que os idosos possam ter uma melhor qualidade de vida, para que vivam o maior tempo possível em suas residências com saúde e independência.

\section{Agradecimentos}

O presente trabalho foi realizado com apoio da Coordenação de Aperfeiçoamento de Pessoal de Nível Superior Brasil (CAPES) - Código de Financiamento 001

\section{Referências}

AGS. American Geriatrics Society. (2015) Updated Beers Criteria for Potentially Inappropriate Medication Use in Older Adults. J Am Geriatr Soc, 63 (11): 2227-2246. 10.1111/jgs.13702.

AGS. American Geriatrics Society. (2019). American Geriatrics Society 2019 Updated AGS Beers Criteria ${ }^{\circledR}$ for Potentially Inappropriate Medication Use in Older Adults. J Am Geriatr Soc, 67(4):674-694. 10.1111/jgs.15767.

Arruda, D. C. J., Eto, F. N., Velten, A. P. C., Morelato, R. L., \& Oliveira E. R. A. (2015). Fatores associados a não adesão medicamentosa entre idosos de um ambulatório filantrópico do Espírito Santo. Rev. Bras. Geriatr. Gerontol, 18(2):327-337 https://doi.org/10.1590/1809-9823.2015.14074

Avorn, J. (2002). Inappropriate prescribing for elderly patients. JAMA. 287(10):1265.

Baldoni, A. de O., Chequer, F. M., Ferraz, E. R., Oliveira D, de P., Pereira, L. R. L., \& Dorta, D. J. (2010). Elderly and drugs: risks and necessity of rational use. Braz. J. Pharm. Sci, 46(4): 617-631. https://doi.org/10.1590/S1984-82502010000400003

Baldoni, A. de O., \& Pereira, L. R. L. (2011). The impact of Brazilian population aging on the health system from the perspective of pharmacoepidemiology: a narrative review. Rev Ciênc Farm Básica Apl, 32(3):313-321.

Baldoni, A. de O., Ayres, L. R., Martinez, E. Z., DewulfI, N. de L., dos Santos, V., Obreli-Neto, P. R., \& Pereira, LR. L. (2013). Pharmacoepidemiological profile and polypharmacy indicators in elderly outpatients. Braz. J. Pharm. Sci, 49(3):443-452. https://doi.org/10.1590/S1984-82502013000300006

Baldoni, A. de O., Ayres. L. R., Martinez, E. Z., Dewulf. N. de L., Dos Santos, V., Pereira, L. R. L. (2014). Factors associated with potentially inappropriate medications use by the elderly according to Beers criteria 2003 and 2012. Int J Clin Pharm, 36(2):316-24. 10.1007/s11096-013-9880-y

Beers, M. H., Ouslander, J. G., Rollingher, I., Reuben, D. B., Brooks, J., \& Beck, J. C. (1991). Explicit criteria for determining inappropriate medication use in nursing home residents. UCLA Division of Geriatric Medicine. Arch Intern Med, 151(9):1825-1832.

Beuscart, J. B., Dupont, C., Defebvre, M. M., \& Puisieux, F. (2014). Potentially inappropriate medications (PIMs) and anticholinergic levels in the elderly: a population based study in a French region. Arch Gerontol Geriatr, 59(3):630-5. 10.1016/j.archger.2014.08.006

Bjorkman, I. K., Fastbomm J., Schmidt, I. K., \& Bernsten, C. B. (2002). Drug-drug interactions in the elderly. Ann Pharmacother, 36(11):1675-1681. 10.1345/aph.1A484

Borja-Oliveira, C. R. (2017). Effect of anticholinergic cognitive burden in the elderly - an integrative review. Estud Interdiscipl Envelhec, 22(2):57-74.

Boustani, M., Campbell, N., Munger, S., Maidment, I., \& Fox, C. (2008). Impact of anticholinergics on the aging brain: a review and practical application. Aging Health, 4(3): 311-320. https://doi.org/10.2217/1745509X.4.3.311

Brunton, 1. 1., Chabner, B. A., Knollmann, B. C. (2018). As Bases Farmacológicas da Terapêutica de Goodman e Gilman. Porto alegre: AMGH.

Campbell, N., Boustani, M., Limbil, T., Ott, C., Fox, C., Maidment, I., Schubert, C. C., Munger, S., Fick, D., Miller, D., \& Gulati, R. (2009). The cognitive impact of anticholinergics: a clinical review. Clin Interv Aging, 4:225-33. 10.2147/cia.s5358

Cardwell, K., Hughes, C. M., \& Ryan, C. (2015). The Association Between Anticholinergic Medication Burden and Health Related Outcomes in the 'Oldest Old': A Systematic Review of the Literature. Drugs Aging, 32(10):835-48. 10.1007/s40266-015-0310-9

Carnahan, R. M., Lund, B. C., Perry, P. J., Pollock, B. G., \& Culp, K. R. (2006). The Anticholinergic Drug Scale as a Measure of Drug-Related Anticholinergic Burden: Associations With Serum Anticholinergic Activity. J Clin Pharmacol, 46(12):1481-1486. 10.1177/0091270006292126

Carvalho, M. F. C., Romano-Lieber, N. S., Bergsten-Mendes, G., Secoli, S. R., Ribeiro, E., Lebrão, M. L. \& Duarte, Y. A. O. (2012). Polifarmácia entre idosos do Município de São Paulo - estudo SABE. Revista Brasileira de Epidemiologia, 15(4): 817-27. https://doi.org/10.1590/S1415-790X2012000400013

Casarin, S. T., Porto, A. R., Gabatz, R. I. B., Bonow, C. A., Ribeiro, J. P., \& Mota, M. S. (2020). Tipos de revisão de literatura: considerações das editoras do Journal of Nursing and Health / Types of literature review: considerations of the editors of the Journal of Nursing and Health. Journal of Nursing and Health, 10(5), 1-7. https://doi.org/10.15210/jonah.v10i5.19924 
Castellar, J. I., Karnikowski, M. G. O., Vianna, L. G., \& Nóbrega, O. T. (2007). Estudo da farmacoterapia prescrita a idosos em instituição brasileira de longa permanência. Acta Med Port, 20: 97-105.

Castilho, E. C., Reis, A. M., Borges, T. L., Siqueira, L. D., \& Miasso, A. (2018). Potential drug-drug interactions and polypharmacy in institutionalized elderly patients in a public hospital in Brazil. J Psychiatr Ment Health Nurs, 25(1):3-13. 10.1111/jpm.12431

Correr, C. J., Pontarolo, R., Ferreira, L. C., \& Baptistão, A. S. (2007). Drug-related problems risks in patients of a geriatric institution. Rev Farm Bioquim Univ Sao Paulo, 43(1):55-62.

Correr, C. J., Otuki, M. F., \& Soler, O. (2011). Assistência farmacêutica integrada ao processo de cuidado em saúde: gestão clínica do medicamento. Revista Pan-Amazônica de Saúde, 2(3).41-9. http://dx.doi.org/10.5123/S2176-62232011000300006

Correr, C. J., \& Otuki, M. F. (2013). A prática farmacêutica na farmácia comunitária. Porto Alegre: Artmed.

Côrrea, J., Lorenz, C., \& Colet, C. F. (2017). Comparação entre estratégias de farmacovigilância hospitalar nos países da américa latina. Revista Contexto \& Saúde, 17(33): 155-166. http://dx.doi.org/10.21527/2176-7114.2017.33.155-166

Costa, R. Me., Lima, V. A. B., Paiva, I. G., Sousa, P. T. P., \& Lima, L. G. (2008). Use of medicines among elderly people. Geriatr Gerontol Aging. 2:126-131.

Costa, S. C., E\& Pedroso, E. R. P. (2011). A prescrição de medicamentos para idosos internados em serviço de clínica médica: atualização. Rev Med Minas Gerais, 21(2): 201-214.

Diaz, R. B. (2002). Adesão ao tratamento medicamentoso em pacientes Idosos. In: M.P. Netto (Ed.), Gerontologia: a velhice e o envelhecimento em visão global (230-241p). São Paulo: Atheneu.

Dimitrow, M. S., Airaksinen, M. S., Kivelä, S. L., Lyles, A., \& Leikola, S. N. (2011). Comparison of prescribing criteria to evaluate the appropriateness of drug treatment in individuals aged 65 and older: a systematic review. J Am Geriatr Soc, 59(8):1521-30. 10.1111/j.1532-5415.2011.03497.x.

Doron, G., Birkan, I., \& Gulistan, B. (2015). Routine deprescribing of chronic medications to combat polypharmacy. Therapeutic advances in drug safety, 6(6):212-233. 10.1177/2042098615613984

Durán, C., AzermaI, M., Vander, \& Stichele, R. (2013). Systematic review of anticholinergic risk scales in older adults. Eur J Clin Pharmacol; 69(7):14851496. $10.1007 / \mathrm{s} 00228-013-1499-3$

Fialová, D., Topinková, E., Gambassi, G., Finne-Soveri, H., Jónsson, P. V., Carpenter, I., Schroll, M., Onder, G., Sorbye, L. W., Wagner, C., Reissigová, J., Bernabei, R., \& AdHOC Project Research Group. (2005). Potentially inappropriate medication use among elderly home care patients in Europe. JAMA, 293(11):1348-58. 10.1001/jama.293.11.1348.

Franceschet-de-Sousa, I., Biscaro, A., Biscaro, F., \& Fernandes, M. S. (2010) Uso Racional de Medicamentos: Relato de Experiência no Ensino Médico da Unesc, Criciúma/SC. Revista Brasileira de Educação Médica, 34 (3) : 438 - 445. https://doi.org/10.1590/S0100-55022010000300014

Freitas, P. E. F., \& Reis, A. M. M. (2018). Prescrição inapropriada de medicamentos a idosos: análise dos critérios publicados de 2011 a 2016. Rev. Bras. Farm. Hosp. Serv. Saúde, 8(4): 31-36. 10.30968/rbfhss.2017.084.006

Fox, C., Smith, T., Maidment, I., Chan, W. Y., Bua, N., Myint, P. K., Boustani, M., Kwok, C. S., Glover, M., Koopmans, I., \& Campbell, N. (2014). Effect of medications with anti-cholinergic properties on cognitive function, delirium, physical function and mortality: a systematic review. Age Ageing, 43(5):604-615. 10.1093/ageing/afu096

Fulton, M. M., \& Allen, E. R. (2005). Polypharmacy in the elderly: a literature review. J Am Acad Nurse Pract, 17(4):123-132. 10.1111/j.10412972.2005.0020.x.

Fustinoni, S., Renard, D., Santos-Eggimann, B., \& Seematter-Bagnoud, L. (2015). Polypharmacy and associated factors among community-dwelling older persons in a Swiss Canton. Clinical Therapeutics, 37(8): e39-e40. https://doi.org/10.1016/j.clinthera.2015.05.119

Gallagher, P. F., Barry, P. J., Ryan, C., Hartigan, I., \& O'Mahony, D. (2008). Inappropriate prescribing in an acutely ill population of elderly patients as determined by Beers' Criteria. Age Ageing, 37 (1): 96-101. 10.1093/ageing/afm116

Galvão, M. P. A., \& Ferreira, M. B. C. (2006). Prescrição de medicamentos em geriatria. In: Fuchs, F.D., Wannmacher, L., Ferreira, M.B.C. (Eds), Farmacologia clínica: fundamentos da terapêutica racional. (3a ed. 1096p), Rio de Janeiro: Guanabara Koogan.

Gerretsen, P., \& Pollock, B. G. (2011). Drugs with anticholinergic properties: a current perspective on use and safety. Expert Opin Drug Saf, 10(5):751-65. $10.1517 / 14740338.2011 .579899$

Gerretsen, P., \& Pollock, B. G. (2013). Cognitive risks of anticholinergics in the elderly. Aging Health, 9(2):159-166. https://doi.org/10.2217/ahe.13.14

Gorzoni, M. L., Fabbri, R. M. A., \& Pires, S. L. (2012). Potentially inappropriate medications in elderly. Revista da Associação Médica Brasileira, 58(4): 442446. https://doi.org/10.1590/S0104-42302012000400014

Gorzoni, M. L., \& Fabri, R. M. (2017). Applicability of Anticholinergic Risk Scale in hospitalized elderly persons. Thematic Section - Drug Use and Associated Risks Among the Elderly. Rev. bras. geriatr. Gerontol, 20(1):123-128. https://doi.org/10.1590/1981-22562017020.150191

Guaraldo, L., Cano, F. G., Damasceno, G. S., \& Rozenfeld, S. (2011). Inappropriate medication use among the elderly: a systematic review of administrative databases. BMC Geriatr, 11:79. 10.1186/1471-2318-11-79

Guthrie, B., Makubate, B., Hernandez-Santiago, V., \& Dreischulte, T. (2015). The rising tide of polypharmacy and drug-drug interactions: Population database analysis 1995-2010. BMC Med, 13(1):74. 10.1186/s12916-015-0322-7 
Gurwitz, J. H., Field, T. S., Harrold, L. R., Rothschild, J., Debellis, K., Seger, A. C., Cadoret, C., Fish, L. S., Garber, L., Kelleher, M., \& Bates, D. W. (2003) Incidence and Preventability of Adverse Drug Events Among Older Persons in the Ambulatory Setting. JAMA, 289(9):1107-16. 10.1001/jama.289.9.1107. PMID: 12622580

Hepler, C. D., \& Strand, L. M. (1990). Opportunities and responsibilities in pharmaceutical care. American Journal of Hospital Pharmacy, 47(3):533-543.

Hori, K., Konishi, K., Tani, M., Tomioka, H., Akita, R., Kitajima, Y., Aoki, M., Yokoyama, S., Azuma, K., Ikuse, D., Akashi, N., Hosoi, M., Jinbo, K., \& Hachisu, M. (2014). Serum anticholinergic activity: a possible peripheral marker of the anticholinergic burden in the central nervous system in Alzheimer's disease. Disease markers, 459013. https://doi.org/10.1155/2014/459013

Hsu, W. H., Wen, Y. W., Chen, L. K., \& Hsiao, F. Y. (2017). Comparative Associations Between Measures of Anti-cholinergic Burden and Adverse Clinical Outcomes. Ann Fam Med, 15(6):561-569. 10.1370/afm.2131

Hilmer, S. N., Mager, D. E., Simonsick, E. M., Ling, S. M., Windham, B. G., Harris, T. B., Shorr, R. I., Bauer, D. C., Abernethy, D. R., \& Health ABC Study. (2009). Drug burden index score and functional decline in older people. Am J Med, 122(12):1142-9 e1-2. 10.1016/j.amjmed.2009.02.021

Institute for Safe Medication Practices Canada. (2018). Deprescribing: Managing Medications to Reduce Polypharmacy. ISMP Canada Safety Bulletin, 18(3):1-6.

Junius-Walker, U., Theile, G., \& Hummers-Pradier, E. (2007). Prevalence and predictors of polypharmacy among older primary care patients in Germany. Family Practice, 24(1):14-19. 10.1093/fampra/cml067

Kashyap, M., Belleville, S., Mulsant, B. H., Hilmer, S. N., Paquette, A., Tu, 1., \& Tannenbaum, C. (2014). Methodological challenges in determining longitudinal associations between anticholinergic drug use and incident cognitive decline. Journal of the American Geriatrics Society, 62(2), 336-341. https://doi.org/10.1111/jgs.12632

Kaufmann, C. P., Tremp, R., Hersberger, K. E., \& Lampert, M. L. (2014). Inappropriate prescribing: a systematic overview of published assessment tools. Eur J Clin Pharmacol. 70(1):1-11. 10.1007/s00228-013-1575-8

Kim, H. A., Shin, J. Y., Kim, M. H., \& Park, B. J. (2014). Prevalence and predictors of polypharmacy among Korean elderly. PLoS One, 9(6): e98043. 10.1371/journal.pone.0098043

Lai, S. W., Liao, K. F., Liao, C. C., Muo, C. H., Liu, C. S., \& Sung, F. C. (2010). Polypharmacy correlates with increased risk for hip fracture in the elderly: a population-based study. Medicine (Baltimore), 89(5):295-309. 10.1097/MD.0b013e3181f15efc

Landi, F., Dell'Aquila, G., Collamati, A., Martone, A.M., Zuliani, G., Gasperini, B., Eusebi, P., Lattanzio, F., \& Cherubini, A. (2014). Anticholinergic drug use and negative outcomes among the frail elderly population living in a nursing home. J Am Med Dir Assoc, 15(11):825-829. 10.1016/j.jamda.2014.08.002.

Lertxundi, U., Domingo-Echaburu, S., Hernandez, R., Peral, J., \& Medrano, J. (2013). Expert-based drug lists to measure anticholinergic burden: similar names, different results. Psychogeriatrics, 13(1):17-24. 10.1111/j.1479-8301.2012.00418.x.

Locatelli, J. (2007). Interações medicamentosas em idosos hospitalizados. Einstein, 5(4):343-346.

Lira, C. B. C., \& de Oliveira, V. M. (2021). A importância da conciliação medicamentosa na prática da farmácia clínica em hospitais. Brazilian Journal of Development, 7(9): 89345-89355. 10.34117/bjdv7n9-207

Lyra Jr, D. P., Oliveira, A. T. C., Lima, K. S. L., Neves, S. J. F., Rocha, C. E., Oliveira, M. A. C., Lima, K. S. Q., Neves, S. J. F., Rocha, C. E., \& Santana, D. P. (2002). Atenção Farmacêutica na dispensação de prescrições médicas. Infarma, 14 (11/12): 43-45.

Lyra Jr, D. P., Prado, M. C. T. A., Abriata, J. P., \& Pelá, I. R. (2004). As Prescriç̃es Médicas como Causadoras de Risco para Problemas Relacionados com os Medicamentos. Seguimento Farmacoterapêutico. Pharmacy Practice, 2(2):86-96.

Lu, C. J., \& Tune, L. E. (2003). Chronic exposure to anticholinergic medications adversely affects the course of Alzheimer disease. Am J Geriatr Psychiatry, 11(4):458- 461

Lu, W. H., Wen, Y. W., Chen, L. K.,\& Hsiao, F. Y. (2015). Effect of polypharmacy, potentially inappropriate medications and anticholinergic burden on clinical outcomes: a retrospective cohort study. CMAJ, 187(4):130-137. 10.1503/cmaj.141219

Lucchetti, G., \& Lucchetti, A. L. (2017). Inappropriate prescribing in older persons: a systematic review of medications available in diferente criteria. Arch Gerontol Geriatr, 68:55-61. 10.1016/j.archger.2016.09.003

Mallet, L., Spinewine, A., \& Huang, A. (2007). The challenge of managing drug interactions in elderly people. Lancet, 370(9582): 185-91. 10.1016/S01406736(07)61092-7

Malta, D. C., Bernal, R. T. I., Lima, M. G., Araújo, S. S. C., Silva, M. M. A., Freitas, M. I. F., \& Barros, M. B. A. (2017). Doenças crônicas não transmissíveis e a utilização de serviços de saúde: análise da Pesquisa Nacional de Saúde no Brasil. Rev Saúde Publica. 2017; 51(1): 1s-4s. https://doi.org/10.1590/S15188787.2017051000090

Marques J. B. V., \& Freitas, D. (2018). Método DELPHI: caracterização e potencialidades na pesquisa em Educação. Revista Pro.Posições, 29 (2): 389-415. https://doi.org/10.1590/1980-6248-2015-0140

Manso, M. E. G., Biffi, E. C. A., \& Gerardi, T. J. (2015). Prescrição inadequada de medicamentos a idosos portadores de doenças crônicas em um plano de saúde no município de São Paulo, Brasil. Rev. Bras. Geriatr. Gerontol, 18(1):151-164. http://dx.doi.org/10.1590/1809-9823.2015.14056 
Marengoni, A., Angleman, S., Melis, R., Mangialasche, F. K. A., Garmen, A., Meinow, B., \& Fratiglioni, L. (2011). Aging with multimorbidity: a systematic review of the literature. Ageing Res Rev, 10(4):430-9. 10.1016/j.arr.2011.03.003.

Mayer, T., Haefeli, W. E., \& Seidling, H. M. (2015). Different methods, different results-how do available methods link a patient's anticholinergic load with adverse outcomes? Eur J Clin Pharmacol, 71(11):1299-314. 10.1007/s00228-015-1932-x

McGrath, K., Hajjar, E. R., Kumar, C., Hwang, C., \& Salzman, B. (2017). Deprescribing: A simple method for reducing polypharmacy. J Fam Pract, 66(7):436-445.

Mercadante, A. C. C., Conti, M. S. B., Wagner, G. A., Andreoni, S. \& Ramos, L. R. (2021). Fatores determinantes da polifarmácia entre idosos residentes em um grande centro urbano da região sudeste do brasil. Revista Valore, 6: 167-182, 2021. https://doi.org/10.22408/reva6020211027167-182

Myint, P. K., Fox, C., Kwok, C. S., Luben, R. N., Wareham, N. J., Khaw, K. T. (2015). Total anticholinergic burden and risk of mortality and cardiovascular disease over 10 years in 21636 middle aged and older men and women of EPIC-Norfolk prospective population study. Age Ageing. 2015; 44: 219-25. 10.1093/ageing/afu185

Nery, R. T., \& Reis, A. M. M. (2019). Desenvolvimento de uma escala brasileira de medicamentos com atividade anticolinérgica. Einstein, 17(2):1-8. 10.31744/einstein_journal/2019AO4435

Oliveira, M. G., Amorim, W. W., Oliveira, C. R. B., Coqueiro, H. L., Gusmão, L. C., \& Passos, L. C. (2016). Consenso Brasileiro De Medicamentos Potencialmente Inapropriados Para Idosos. Geriatr Gerontol Aging, 4:1-14.

O'Mahony, D., O'Sullivan, D., Byrne, S., O'Connor, M. N., Ryan, C., \& Gallagher, P. (2015). STOPP/START criteria for potentially inappropriate prescribing in older people: version 2. Age Ageing, 44(2):213-218. 10.1093/ageing/afu145.

Onder, G., Pedone, C., Landi, F., Cesari, M., Della Vedova, C., Bernabei, R., \& Gambassi, G. (2002). Adverse drug reactions as cause of hospital admissions: results from the Italian Group of Pharmacoepidemiology in the Elderly (GIFA). J Am Geriatr Soc, 50(12):1962-1968. 10.1046/j.1532-5415.2002.50607.x.

Onder, G., Landi, F., Cesari, M., Gambassi, G., Carbonin, P., Bernabei, R., \& Investigators of the GIFA Study. (2003). Inappropriate medication use among hospitalized older adults in Italy: results from the Italian Group of Pharmacoepidemiology in the elderly. Eur J Clin Pharmacol. 59 (2): 157-62. $10.1007 / \mathrm{s} 00228-003-0600-8$

Passarelli, M. C., Jacob-Filho, W., \& Figueras, A. (2005). Adverse drug reactions in an elderly hospitalised population: inappropriate prescription is a leading cause. Drugs Aging, 22(9):767-77. 10.2165/00002512-200522090-00005

Pasina, L., Djade, C. D., Lucca, U., Nobili, A., Tettamanti, M., Franchi, C., Salerno, F., Corrao, S., Marengoni, A., Iorio, A., Marcucci, M., Violi, F. \& Mannucci, P. M. (2013). Association of anticholinergic burden with cognitive and functional status in a cohort of hospitalized elderly: comparison of the anticholinergic cognitive burden scale and anticholinergic risk scale: results from the REPOSI study. Drugs Aging, 30(2):103-112. 10.1007/s40266-012-0044-

Penteado, P. T. P. S., Cunico, C., Oliveira, K. S., Polichuk, M. O. (2002). O uso de Medicamentos por idosos. Visão Acadêmica, 3(1): 35-42. http://dx.doi.org/10.5380/acd.v3i1.498

Pereira, L. R. L., Vecchi, L. U. P., Baptista, M. E. C., \& Carvalho, D. (2004). Avaliação da utilização de medicamentos em pacientes idosos por meio de conceitos de farmacoepidemiologia e farmacovigilância. Ciência \& Saúde Coletiva, 9(2): 479-481. https://doi.org/10.1590/S1413-81232004000200023

Pereira, K. G., Peres, M. A., Iop, D., Boing, A. C., Boing, A. F., Aziz, M., \& d’Orsi, E. (2017). Polifarmácia em idosos: um estudo de base populacional. Rev Bras Epidemiol, 20(2):335-344. https://doi.org/10.1590/1980-5497201700020013

Persaud, M., Holroyd-Leduc, J. M. (2014). Anticholinergic medications in the older adult: A hidden burden. Journal of the Canadian Geriatrics Society, 4(2): 4-7.

Pont, L. G., Nielen, J. T., McLachlan, A., Gnjidic, D., \& Chan, L. (2015). Cumming RG, Taxis K. Measuring anticholinergic drug exposure in older community-dwelling Australian men: a comparison of four different measures. Br J Clin Pharmacol, 80(5):1169- 1175. 10.1111/bcp.12670

Portela, A. S., Simões, M. O. S., Fook, S. M. L., Montenegro Neto, A. N., \& Silva, P. C. D. (2010). Prescrição médica: orientações adequadas para o uso de medicamentos? Ciência \& Saúde Coletiva, 15(3):3523-3528. https://doi.org/10.1590/S1413-81232010000900027

Praxedes, M. F. S., Telles, P. C. P., \& Filho Pinheiro, M. L. P. (2011). Identificação e análise de prescrições de medicamentos potencialmente inapropriados para idosos em uma Instituição Hospitalar. Cienc Cuid Saúde, 10(2):338-44. https://doi.org/10.4025/ciencuidsaude.v10i2.10214

Praxedes, M. F. S., Pereira, G. C. S., Lima, C. F. M., Santos, D. B., \& Berhends, J. S. (2021). Prescrição de medicamentos potencialmente inapropriados para idosos segundo os Critérios de Beers: revisão sistemática. Ciência \& Saúde Coletiva, 26(8):3209-3219. 10.1590/1413-81232021268.05672020

Rang, H. P., Dale, M. M., Flower, R. J., \& Henderson, G. (2012). Rang \& Dale's Pharmacology. Elsevie.

Ramos, L. R. (2003). Determinant factors for healthy aging among senior citizens in a large city: the Epidoso Project in São Paulo. Cad. Saúde Pública, 19 (3): 793-798. https://doi.org/10.1590/S0102-311X2003000300011

Ramos, L. R., Tavares, N. U. L., Bertoldi, A. D., Farias, M. R., Oliveira, M. A., Luiza, V. L., Dal Pizzol, T. S., Arrais, P. S. D., \&Mengue, S. S. (2016). Polypharmacy and Polymorbidity in Older Adults in Brazil: a public health challenge. Rev Saúde Pública, 50(2):1s-9s. https://doi.org/10.1590/S15188787.2016050006145

Reich, O., Rosemann, T., Rapold, R., Blozik, E., \& Senn, O. (2014). Potentially Inappropriate Medication Use in Older Patients in Swiss Managed Care Plans: Prevalence, Determinants and Association with Hospitalization. PLoS One, 9(8):23-5. 10.1371/journal.pone.0105425. 
Research, Society and Development, v. 11, n. 2, e55311225942, 2022

Ribeiro, A. Q., Araújo, C. M. C., Acurcio, F. A., Magalhães, S. M. S., \& Chaimowicz, F. (2005). Qualidade do uso de medicamentos por idosos: uma revisão dos métodos de avaliação disponíveis. Ciência \& Saúde Coletiva, 10 (4): 1037-45. https://doi.org/10.1590/S1413-81232005000400026

Rocha, C. H., Oliveira, A. P. S., Ferreira, C., Faggiani, F. T., Schroeter, G., Souza, A. C. A., DeCarli, G. A., Morrone, F. B., \& Werlang, M. C. (2008). Adesão à prescrição médica em idosos de Porto Alegre, RS. Revista Ciência \& Saúde Coletiva, 13: 702-710. https://doi.org/10.1590/S1413-81232008000700020

Rochon, P. A., \&Gurwitz, J. H. (1995). Drug therapy. Lancet, 346 (8966): 32-6. 10.1016/s0140-6736(95)92656-9

Rochon, P. A., Petrovic, M., Cherubini, A., Onder, G., O’Mahony, D., Sternberg, S. A., Stall, N. M., \& Gurwitz, J. H. (2021). Polypharmacy, inappropriate prescribing, and deprescribing in older people: through a sex and gender lens. Lancet Healthy Longev, 2(5): E290 -E300. https://doi.org/10.1016/S2666$7568(21) 00054-4$

Rothberg, M. B., Pekow, P. S., Liu, F., Korc-Grodzicki, B., Brennan, M. J., Bellantonio, S., Heelon, M., \& Lindenauer, P. K. (2008). Potentially Inappropriate Medication Use in Hopitalized Elders. J Hosp Med, 3(2):91-102. 10.1002/jhm.290

Rother, E. T. (2007). Revisão sistemática X revisão narrativa. Acta paul. Enferm, 20 (2). https://doi.org/10.1590/S0103-21002007000200001

Rovers, J. P., \& Currie, J. D. (2010). Guia Prático da Atenção Farmacêutica: Manual de Habilidades Clínicas. São Paulo: Pharmabooks.

Rozenfeld, S. (2003). Prevalência, fatores associados e mau uso de medicamentos entre os idosos: uma revisão. Cadernos de Saúde Pública, 19(3): 717-724. https://doi.org/10.1590/S0102-311X2003000300004

Rudd, K. M., Raehl, C. L., Bond, C. A., Abbruscato, T. J., \& Stenhouse, A. C. (2005). Methods for assessing drug-related anticholinergic activity. Pharmacotherapy, 25(11):1592-601. 10.1592/phco.2005.25.11.1592

Rudolph, J. L., Salow, M. J., Angelini, M. C., \& McGlinchey, R. E. (2008). The anticholinergic risk scale and anticholinergic adverse effects in older persons. Arch Intern Med, 168(5):508-513. 10.1001/archinternmed.2007.106

Ruxton, K., Woodman, R., \& Mangoni, A. (2015). Drugs with anticholinergic effects and cognitive impairment, falls and all-cause mortality in older adults: A systematic review and meta- analysis. Br J Clin Pharmacol, 80(2):209-220. 10.1111/bcp.12617

Salahudeen, M. S., Duffull, S. B., \& Nishtala, P. S. (2015). Anticholinergic burden quantified by anticholinergic risk scales and adverse outcomes in older people: a systematic review. BMC Geriatrics, 15(31):1-14. 10.1186/s12877-015-0029-9

Scott, I., \& Jayathissa, S. (2010). Quality of drug prescribing in older patients: is there a problem and can we improve it? Intern Med J, 40(1):7-18. 10.1111/j.1445-5994.2009.02040.x.

Secoli, S. R. (2010). Polifarmácia: interações e reações adversas no uso de medicamentos por idosos. Rev Bras Enferm, 63(1): 136-40. https://doi.org/10.1590/S0034-71672010000100023

Shah, B. M., \& Hajjar, E. R. (2012). Polypharmacy, adverse drug reactions, and geriatric syndromes. Clin Geriatr Med, 28(2):173-86. 10.1016/j.cger.2012.01.002

Shekelle, P. G., MacLean, C. H., Morton, S. C., \& Wenger, N. S. (2001). Acove quality indicators. Ann Intern Med, 135(8 Pt 2):653-667. 10.7326/0003-4819135-8_part_2-200110161-00004

Silva, A. L., Ribeiro, A. Q., Klein, C. H. \& Acurcio, F. A. (2012). Utilização de medicamentos por idosos brasileiros, de acordo com a faixa etária: um inquérito postal. Cad. Saúde Pública, 28(6):1033-1045. https://doi.org/10.1590/S0102-311X2012000600003

Silva, R. N. P., Santos, T. G. O., \& Lima, J. A. V. (2018). Uso racional de medicamentos: vantagens, desafios e perspectivas. Rev. Saberes daFSP/SP. 8 (2): 1 10 .

Sousa-Muñoz, R. L., Ibiapina, G. R., Gadelha, C. S., \& Maroja, J. L. S. (2012). Prescrições geriátricas inapropriadas e polifarmacoterapia em enfermarias de clínica médica de um Hospital-Escola. Rev. Bras. Geriatr. Gerontol, 15(2):315-323. https://doi.org/10.1590/S1809-98232012000200014

Spinewine, A., Schmader, K. E., Barber, N., Hughes, C., Lapane, K. L., Swine, C., \& Hanlon, J. T. (2007). Appropriate prescribing in elderly people: how well can it be measured and optimized? Lancet, ;370 (9582):173-184. 10.1016/S0140-6736(07)61091-5

Steinman, M. A., \& Hanlon, J. T. (2010). Managing medications in clinically complex elders: "There's got to be a happy medium". JAMA, 304 (14):1592-601. 10.1001/jama.2010.1482

Vasconcelos, A. M. N., \& Gomes, M. M. F. (2012). Transição demográfica: a experiência brasileira. Epidemiologia e Serviços de Saúde, 21(4): 539-548. https://dx.doi.org/10.5123/S1679-49742012000400003

Veras, R. (2009). Envelhecimento populacional contemporâneo: demandas, desafios e inovações. Rev. Saúde Pública, 43 (3): 548-554. https://doi.org/10.1590/S0034-89102009005000025

Veras, R. P. \& Oliveira, M. (2018). Envelhecer no brasil: A construção de um modelo de cuidado. Revista Ciência \& Saúde Coletiva, 23 (6): 1929-1936. $10.1590 / 1413-81232018236.04722018$

Vuyk, J. (2003). Pharmacodynamics in the elderly. Best Pract Res Clin Anaesthesiol, 17(2):207-218. 10.1016/s1521-6896(03)00008-9.

Teixeira, J. J. V., \& Lefévre, F. (2001). Drug prescription from the perspective of elderly patients. Rev Saúde Pública, 35(2):207-213. https://doi.org/10.1590/S0034-89102001000200016.

Yamagata, A. T., Júnior, R. M. C. B., Galato, D., Meiners, M. M. M. A., \& Silva, E. V. (2018). Profile of studies of potential drug interactions in brazilian hospitals: an integrative review of the literature. Rev Bras Farm Hosp Serv Saude, 9(4): 1-9. 10.30968/rbfhss.2018.094.003 
Research, Society and Development, v. 11, n. 2, e55311225942, 2022

(CC BY 4.0) | ISSN 2525-3409 | DOI: http://dx.doi.org/10.33448/rsd-v11i2.25942

Wan-Hsuan, L., Yu-Wen, W., Liang-Kung, C., \& Fei-Yuan, H. (2015). Effect of polypharmacy, potentially inapropriate medications and anticholinergic burden on clinical outcomes: a retrospective cohort study. CMAJ, 187(4): 1369-1376. 10.1503/cmaj.141219.

Wannmacher, L. (2006). Medicamentos essenciais: vantagens de trabalhar com este contexto. Organização Pan-Americana da Saúde (OPAS): Uso racional de medicamentos. Temas selecionados, 3 (2): 1-6. Retrieved:2021, from: https://www.paho.org/bra/dmdocuments/HSE_URM_MEE_0106.pdf

Wawruch, M., Zikavska, M., Wsolova, L., Kuzelova, M., Tisonova, J., Gajdosik, J., Urbanek,K., \& Kristova, V. (2008). Polypharmacy in elderly hospitalised patients in Slovakia. Pharm World Sci, 30 (3): 235-42. 10.1007/s11096-007-9166-3

Wehling, M. (2009). Multimorbidity and polypharmacy: how to reduce the harmful drug load and yet add needed drugs in the elderly? Proposal of a new drug classification: fit for the aged. J Am Geriatr Soc, 57(3):560-1.

Willcox, S. M., Himmelstein, D. U., \& Woolhandler, S. (1994). Inappropriate drug prescribing for the community-dwelling elderly. JAMA, 272(4):292-6.

World Health Organization. (1987). The Rational Use of Drugs. Report of the Conference of Experts. World Health Organization [WHO]. Retrieved 2021, from: https://apps.who.int/medicinedocs/en/m/abstract/Js17054e/

World Health Organization. (2002). Promoción del uso racional de medicamentos: componentes centrales Perspectivas Politicas sobre medicamentos en la OMS. World Health Organization [WHO]. Retrieved: 2021, from: https://apps.who.int/medicinedocs/en/d/Js4874s/ 